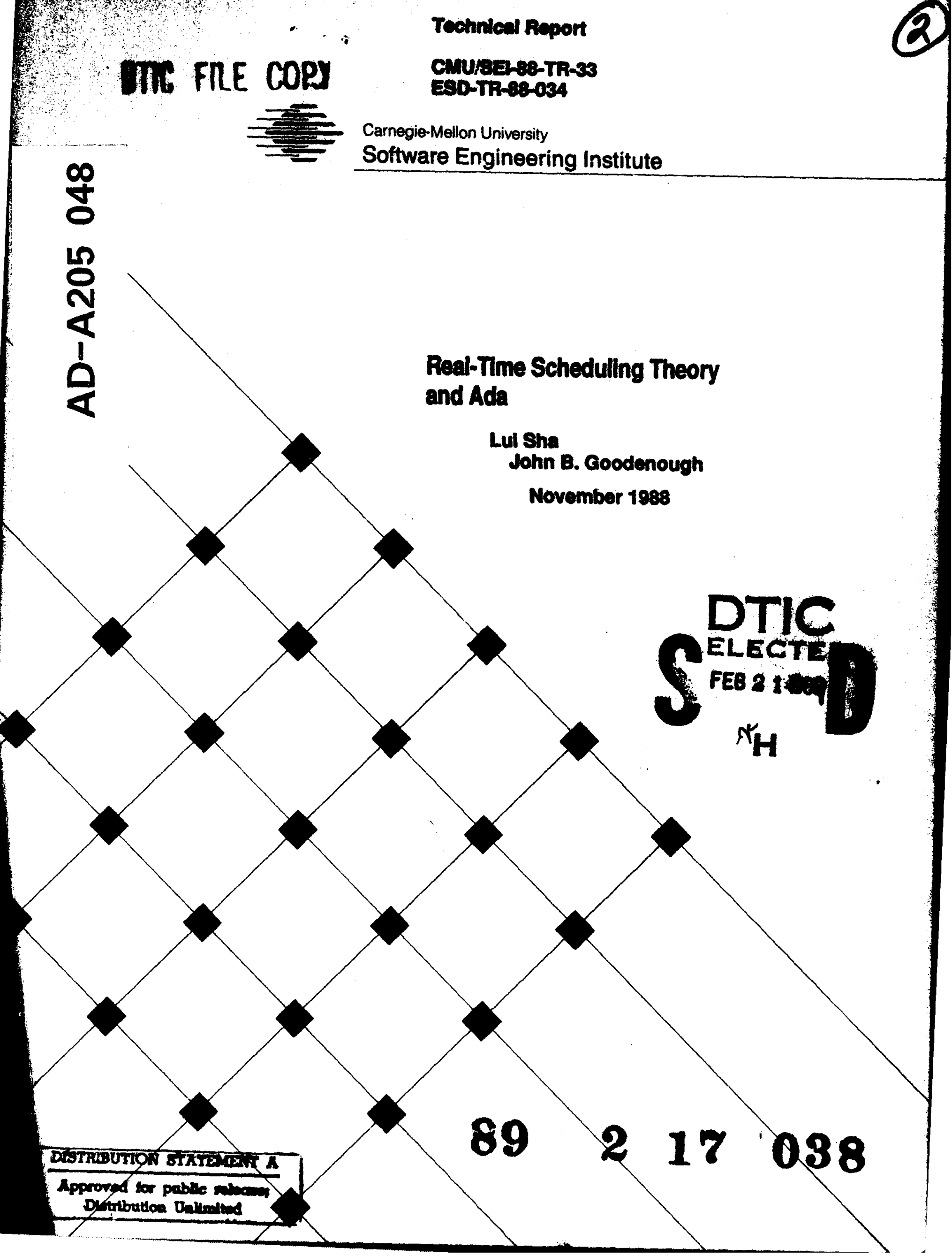


Technical Report

CMU/SEI-88-TR-33

ESD-TR-88-034

November 1988

Real-Time Scheduling Theory and Ada 돌

Lui Sha John B. Goodenough

Real-Time Scheduling in Ada Project 
This technical report was prepared for the

\section{SEl Joint Program Office ESD/AVS \\ Hanscom AFB, MA 01731}

The ideas and findings in this report should not be construed as an official DoD position. It is published in the interest of scientific and technical information exchange.

\section{Revlew and Approval}

This report has been reviewed and is approved for publication.

\section{FOR THE COMMANDER}

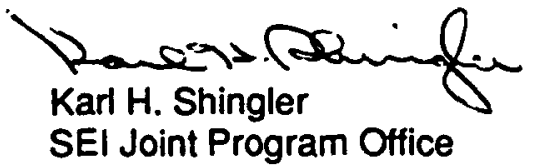

This work is sponsored by the U.S. Department of Defense.

Copyright $\odot 1988$ by Carnegie Mellon University.

This document is available through the Defense Tectmical twonmation Center. DTIC provides access 10 and transler of scientific and rechnical information for DOD personnel, DOD contractors and potential contractors, and other U.S. Government agency perconnel and their contractors. To obtain a copy, please contact DTIC directly: Delense Technical Information Center, Aten: FDPA, Cameron Station, Alexandria, VA 22304-6145.

Copies of this document are also available through the National Technical Inlormation Service. For information on ordering. please contact NTIS directly: National Technical Information Service. U.S. Department of Commerce. Springfield. VA 22161.

The use of any trademark in this publication is not intended in any way to infringe on the rights of the trademark holder. Poviewed and edited by Information Management, a function of the Technology Transition Program, Soltware Engineering Institute. 


\section{Table of Contents}

1. Introduction

1.1. Background

1.2. Controlling Priority Inversion

2. Scheduling Real-Time Tasks

2.1. Periodic Tasks

2.2. Stability Under Transient Overload

2.3. Scheduling Both Aperiodic and Periodic Tasks

2.4. Task Synchronization

2.5. An Example Application of the Theory

3. Real-Time Scheduling in Ada

3.1. On Ada Scheduling Rules

3.2. Ada Real-Time Design Guidelines

4. Conclusion

References

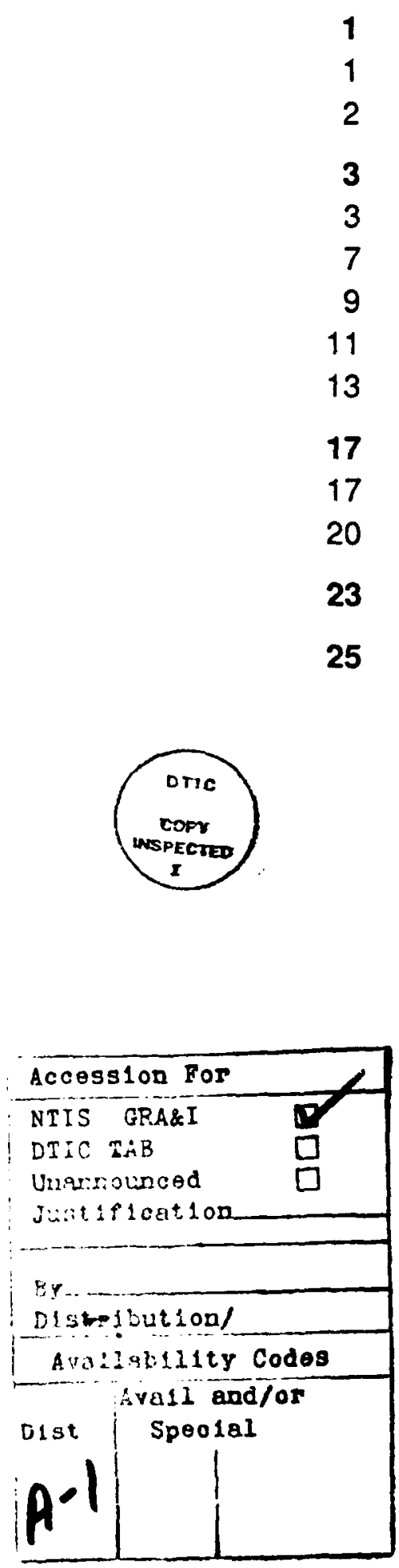




\section{List of Figures}

Figure 2-1: Scheduling Both Aperiodic and Periodic Tasks

Figure 3-1: Blocking Due to the Priority Ceiling Protocol 


\section{List of Tables}

Table 2-1: Scheduling Bounds for One to Nine Independent Tasks

Table 2-2: Minor Cycle Timeline: Each minor cycle is 50. 


\title{
Real-Time Scheduling Theory and Ada
}

\begin{abstract}
The Ada tasking model was intended to facilitate the management of concurrency in a priority-driven scheduling environment. In this paper, we will review some important results of a priority-based scheduling theory, illustrate its applications with examples, discuss its implications for the Ada tasking model, and suggest workarounds that permit us to implement analytical scheduling algorithms within the existing framework of Ada.
\end{abstract}

\section{Introduction}

\subsection{Background}

Traditionally, many real-time systems use cyclical executives to schedule concurrent threads of execution. Under this approach, a programmer lays out the execution timeline by hand to serialize the execution of critical sections and to meet task deadlines. While such an approach is adequate for simple systems, it quickly becomes unmanageable for large systems. It is a painful process to iteratively divide high level language code so the compiled machine code segments can be fitted into time slots of a cyclical executive and that critical sections of different tasks do not interleave. Forcing programmers to schedule tasks by fitting machine code slices on a timeline is no better than the outdated approach of managing memory by manual memory overlay. Such an approach often destroys program struc-

ture and results in real-time programs that are difficult to understand and maintain.

The Ada tasking model represents a fundamental departure from the cyclical executive model. Indeed, the dynamic preemption of tasks at runtime generates non-deterministic timelines that are at odds with the very idea of the fixed execution timeline required by a cyclical executive. From the viewpoint of real-time scheduling theory, an Ada task represents a concurrent unit for scheduling. As long as the real-time scheduling algorithms are supported by the Ada runtime and the resource utilization bounds on CPU, VO drivers, and communication media are observed, the timing constraints will be guaranteed. Even if there is a transient overload, a fixed subset of critical tasks can still meet their deadlines as long as they are schedulable by themselves. In other words, the integration of Ada tasking with analytical scheduling algorithms allows programmers to meet timing constraints by managing resource requirements and relative task importance. This makes Ada tasking truly useful for real-time applications while also making real-time systerns easier to develop and maintain.

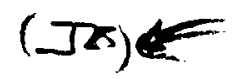




\subsection{Controlling Priority Inversion}

To put real-time scheduling on an analytical basis, systems must be built in a way that ensures that high-priority tasks are minimally delayed by lower priority tasks when both are contending for the same resources. Priority inversion occurs when the use of a resource by a low priority task delays the execution of a high priority task. Priority inversion occurs either when task priorities are incorrectly assigned or when they are not used correctly when allocating resources. One common mistake in priority scheduling is assigning priorities solely according to task importance.

Example 1: Suppose that $\tau_{1}$ and $\tau_{2}$ are periodic tasks with periods 100 and 10 , respectively. Both of them are initiated at $t=0$, and task $\tau_{1}$ is more important than task $\tau_{2}$. Assume that task $\tau_{1}$ requires 10 units of execution time and its first deadline is at $t=100$, while task $\tau_{2}$ needs 1 unit of execution time with its first deadline at $t=10$. If task $\tau_{1}$ is assigned higher scheduling priority because of its importance, task $\tau_{2}$ will miss its deadline unnecessarily even though the total processor utilization is only 0.2 . Both tasks can meet their deadlines using the rate monotonic algorithm [5], which assigns higher priorities to tasks with shorter periods. In fact, many more new tasks can be added into the system by using the simple rate monotonic scheduling algorithm.

Although priority inversion is undesirable, it cannot be completely eliminated. For example, when a low priority task is in a critical region, the higher priority task that needs the shared data must wait. Nonetheless, the duration of priority inversion must be tightly bounded in order to ensure a high degree of responsiveness and schedulability. Controlling priority inversion is a system level problem. The tasking model, runtime support, program design, and hardware architecture should all be part of the solution, not part of the problem. For example, there is a serious priority inversion problem in some existing IEEE 802.5 token ring implementations. While there are 8 priority levels in the token arbitration, the queueing of message packets is FIFO, i.e., message priorities are ignored. As a result, when a high priority packet is behind a low priority packet, the high priority packet has to wait not only for the lower priority packet to be transmitted, but also for the transmission of all medium priority packets in the network. The result is "hurry-up at the processor but miss the deadline at the communication network." Using FIFO queueing in a real-time system is a classical case of priority inversion and can lead to extremely poor schedulability. Priority assignments must be observed at every level of a system for all forms of resource allocation. Minimizing the duration of priority inversion is the key to meeting deadlines and keeping systems responsive to aperiodic events.

Section 2 reviews some of the important results in real-time scheduling theory. We begin with the problem of scheduling independent periodic tasks. Next, we address the issues of maintaining stability under transient overload and the problem of scheduling both periodic and aperiodic tasks. We conclude Section 2 by reviewing the problems of real-time synchronization. In Section 3, we review the Ada tasking scheduling model and suggest some workarounds that permit us to implement many of the scheduling algorithms within the framework of existing Ada rules. Finally, we conclude this paper in Section 4. 


\section{Scheduling Real-Time Tasks}

In this section, we provide an overview of some of the important issues of a real-time scheduling theory. We will begin with the problem of ensuring that independent periodic tasks meet their deadlines. Next, we show how to ensure that critical tasks meet their deadlines even when a system is temporarily overloaded. We then address the problem of scheduling both periodic and aperiodic tasks. Finally, we conclude this section by reviewing the problems of real-time synchronization and communication.

\subsection{Periodic Tasks}

Tasks are independent if their executions need not be synchronized. Given a set of independent periodic tasks, the rate monotonic scheduling algorithm gives each task a fixed priority and assigns higher priorities to tasks with shorter periods. A task set is said to be schedulable if all its deadlines are met, i.e., if every periodic task finishes its execution before the end of its period. Any set of independent periodic tasks is schedulable by the rate monotonic algorithm if the condition of Theorem 1 is met [5].

Theorem 1: A set of $n$ independent periodic tasks scheduled by the rate monotonic algorithm will always meet its deadlines, for all task phasings, if

$$
\frac{C_{1}}{T_{1}}+\cdots+\frac{C_{n}}{T_{n}} \leq n\left(2^{1 / n}-1\right)=U(n)
$$

where $C_{i}$ and $T_{i}$ are the execution time and period of task $\tau_{i}$ respectively.

Theorem 1 offers a sufficient (worst-case) condition that characterizes the schedulability of the rate monotonic algorithm. This bound converges to $69 \%(\ln 2)$ as the number of tasks approaches infinity. Table 2-1 shows values of the bound for one to nine tasks.

$$
\begin{array}{lll}
U(1)=1.0 & U(4)=0.756 & U(7)=0.728 \\
U(2)=0.828 & U(5)=0.743 & U(8)=0.724 \\
U(3)=0.779 & U(6)=0.734 & U(9)=0.720
\end{array}
$$

Table 2-1: Scheduling Bounds for One to Nine Independent Tasks

The bound of Theorem 1 is very pessimistic because the worst-case task set is contrived and unlikely to be encountered in practice. For a randomly chosen task set, the likely bound is $88 \%$ [3]. To know if a set of given tasks with utilization greater than the bound of Theorem 1 can meet its deadlines, the conditions of Theorem 2 must be checked [3].

Theorem 2: A set of $n$ independent periodic tasks scheduled by the rate monotonic algorithm will always meet its deadlines, for all task phasings, if and only if 


$$
\forall i, 1 \leq i \leq n, \quad \min _{(k, I) \varepsilon R_{i}} \sum_{j=1}^{i} C_{j} \frac{1}{l T_{k}}\left\lceil\mid \frac{I T_{k}}{T_{j}}\right\rceil \leq 1
$$

where $C_{j}$ and $T_{j}$ are the execution time and period of task $\tau_{j}$ respectively and $R_{i}=\left\{(k, l) \mid 1 \leq k \leq i, l=1, \cdots,\left\lfloor T_{i} T_{k}\right\rfloor\right\}$.

This theorem provides the exact schedulability criterion for independent periodic task sets under the rate monotonic algorithm. In effect, the theorem checks if each task can complete its execution before its first deadline by checking all the scheduling points. ${ }^{1}$ The scheduling points for task $\tau$ are $\tau$ 's first deadline and the ends of periods of higher priority tasks within $\tau$ 's first deadline. In the formula, $i$ denotes the task to be checked and $k$ denotes each of the tasks that affects the completion time of task $i$, i.e., task $i$ and the higher priority tasks. For a given $i$ and $k$, each value of $l$ represents the scheduling points of task $k$. For example, suppose that we have tasks $\tau_{1}$ and $\tau_{2}$ with periods $T_{1}=5$ and $T_{2}=14$. For task $\tau_{i}(i=1)$ we have only one scheduling point, the end of task $\tau_{1}$ 's first period, i.e., $i=k=1$ and $\left(l=1, \cdots,\left\lfloor T_{i} / T_{k}\right\rfloor=\left\lfloor T_{1} / T_{1}\right\rfloor=1\right)$. The scheduling point is, of course, $\tau_{1}$ 's first deadline $\left(l T_{k}=5, l=1, k=1\right)$. For task $\tau_{i}(i=2)$, there are two scheduling points from all higher priority tasks, $\tau_{k}(k=1)$, i.e., $\left(l=1, \cdots,\left\lfloor T_{i} / T_{k}\right\rfloor=\left\lfloor T_{2} / T_{1}\right\rfloor=2\right)$. The two scheduling points are, of course, the two end points of task $\tau_{1}$ 's period within the first deadline of task $\tau_{2}$ at 14, i.e., $\left(l T_{k}=5, l=1, k=1\right)$ and $\left(l T_{k}=10, l=2, k=1\right)$. Finally, there is the scheduling point from $\tau_{2}$ 's own first deadline, i.e., $\left(l T_{k}=14, l=1, k=2\right)$. At each scheduling point, we check if the task in question can complete its execution at or before the scheduling point. This is illustrated in detail by Examples 3 and 8 below.

Example 2: Consider the case of three periodic tasks, where $U_{i}=C_{i} / T_{i}$.

- Task $\tau_{1}: C_{1}=20 ; T_{1}=100 ; U_{1}=0.2$

- Task $\tau_{2}: C_{2}=40 ; T_{2}=150 ; U_{2}=0.267$

- Task $\tau_{3}: C_{3}=100 ; T_{3}=350 ; U_{3}=0.286$

The total utilization of these three tasks is 0.753 , which is below Theorem 1 's bound for three tasks: $3\left(2^{1 / 3}-1\right)=0.779$. Hence, we know these three tasks are schedulable, i.e., they will meet their deadlines if $\tau_{1}$ is given the highest priority, $\tau_{2}$ the next highest, and $\tau_{3}$ the lowest.

The remaining $24.7 \%$ processor capacity can be used for low priority background processing. However, we can also use it for additional hard real-time computation.

Example 3: Suppose we replace $\tau_{1}$ 's algorithm with one that is more accurate and computationally intensive. Suppose the new algorithm doubles $\tau_{1}$ 's computation time from 20 to 40 . so the total processor utilization increases from 0.753 to 0.953 . Since the utilization of the

\footnotetext{
'Il was shown in [5] that when all the tasks are initiated at the same time (the worst-case phasing), if a task completes its execution before the end of tis first period, it will never miss a deadline.
} 
first two tasks is 0.667 , which is below Theorem 1's bound for two tasks, $2\left(2^{1 / 2}-1\right)=0.828$, the first two tasks cannot miss their deadlines. For task $\tau_{3}$, we use Theorem 2 to check whether the task set is schedulable, i.e., we set $i=n=3$, and check whether one of the following equations holds:

$$
\forall k, l, 1 \leq k, l \leq 3, \quad \sum_{j=1}^{3}\left\lceil\frac{l T_{k}}{T_{j}}\right\rceil C_{j} \leq l T_{k}
$$

To check if task $\tau_{3}$ can meet its deadline, it is only necessary to check the equation for values of $l$ and $k$ such that $l T_{k} \leq T_{3}=350$. If one of the equations is satisfied, the task set is schedulable.

$$
\begin{array}{llll} 
& C_{1}+C_{2}+C_{3} \leq T_{1} & 40+40+100>100 & l=1, k=1 \\
\text { or } & 2 C_{1}+C_{2}+C_{3} \leq T_{2} & 80+40+100>150 & l=1, k=2 \\
\text { or } & 2 C_{1}+2 C_{2}+C_{3} \leq 2 T_{1} & 80+80+100>200 & l=2, k=1 \\
\text { or } & 3 C_{1}+2 C_{2}+C_{3} \leq 2 T_{2} & 120+80+100=300 & l=2, k=2, \text { or } l=3, k=1^{2} \\
\text { or } & 4 C_{1}+3 C_{2}+C_{3} \leq T_{3} & 160+120+100>350 & l=1, k=3
\end{array}
$$

The analysis shows that task $\tau_{3}$ is also schedulable and in the worst-case phasing will meet its deadline exactly at time 300 . Hence, we can double the utilization of the first task from $20 \%$ to $40 \%$ and still meet all the deadlines. The remaining $4.7 \%$ processor capacity can be used for either background processing or a fourth hard deadline task, which has a period longer than that of $\tau_{3}{ }^{3}$ and which satisfies the condition of Theorem 2.

A major advantage of using the rate monotonic algorithm is that it allows us to separate logical correctness concerns from timing correctness concerns. Suppose that a cyclical executive is used for this example. The major cycle must be the least common multiple of the task periods. In this example, the task periods are in the ratio 100:150:350 =2:3:7. A minor cycle of 50 units would induce a major cycle of 42 minor cycles, which is an overly complex design. To reduce the number of minor cycles, we can try to modify the periods. For example, it might be possible to reduce the period of the longest task, from 350 to 300 . The total utilization is then exactly $100 \%$, and the period ratios are 2:3:6; the major cycle can then be 5 minor cycles of 50 units. To implement this approach and minimize the splitting of computations belonging to a single task, we could split task $\tau_{1}$ into two parts of 20 units computation each, $C_{1,1}$ and $C_{1,2}$. The computation of task $\tau_{2}$ similarly could be split into at least two parts such that task $\tau_{3}$ need only be split into four parts. A possible timeline in-

\footnotetext{
${ }^{2}$ That is, after 300 units of time, $\tau_{1}$ will have nu three times, $\tau_{2}$ will have run twice, and $\tau_{3}$ will have run once. The required amount of computation just fits within the allowed time, so each task meets its deadline. [5] showed that since the tasks meet their deadlines at least once within the period $T_{3}$, they will ahways meet their deadlines.

${ }^{3}$ Task $\tau_{3}$ just meets its deadline at 300 and hence we cannot add a task with a priority higher than that of task 3.
} 
dicating the amount of computation for each task in each minor cycle is shown in the following table, where $20_{1}$ on the first line indicates the first part of task $\tau_{1}$ 's computation, which takes 20 units of time.

\begin{tabular}{|c|c|c|c|c|c|c|}
\hline \multicolumn{6}{|c|}{ Cyclic Timeline for Example 3 } \\
\hline & 1 & 2 & 3 & 4 & 5 & 6 \\
\hline$\tau_{1}$ & $20_{1}$ & $20_{2}$ & $20_{1}$ & $20_{2}$ & $20_{1}$ & $20_{2}$ \\
\hline$\tau_{2}$ & $30_{1}$ & $10_{2}$ & & $30_{1}$ & $10_{2}$ & \\
\hline$\tau_{3}$ & & $20_{1}$ & $30_{2}$ & & $20_{3}$ & $30_{4}$ \\
\hline
\end{tabular}

Table 2-2: Minor Cycle Timeline: Each minor cycle is 50.

When processor utilization level is high and there are many tasks, fitting code segments into time slots can be a time-consuming iterative process. In addition, later modification of any task may overflow a particular minor cycle and require the entire timeline to be redone. But more important, the cyclic executive approach has required us to modify the period of one of the tasks, increasing the utilization to $100 \%$ without in fact doing more useful work. Under the rate monotonic approach, all deadlines are met, but machine utilization is $95.3 \%$ instead of $100 \%$. This doesn't mean the rate monotonic approach is less efficient. The capacity that isn't needed to service real-time tasks can be used by background tasks, e.g., for builtin-test purposes. With the cyclic executive approach, no such additional work can be done in this example. Of course, the scheduling overhead for preemptive scheduling needs to be taken into account. If $S$ is the amount of time needed for a single scheduling action, then since there are two scheduling actions per task, the total utilization devoted to scheduling is $2 S / T_{1}+2 S / T_{2}+2 S / T_{3}$. In the cyclic case, the scheduling overhead is partly in the time needed to dispatch each task's code segment in each minor cycle and partly in the utilization wasted by decreasing the period for task 3 . For this example, the scheduling overhead for the cyclic approach is at least $4.7 \%$ :

$$
\begin{aligned}
& \text { Actual_Utilization - Required_Utilization, i.e., } \\
& 100 / 300-100 / 350=.333-.286=.047
\end{aligned}
$$

Thus, although the rate monotonic approach may seem to yield a lower maximum utilization than the cyclic approach, in practice, the cyclic approach may simply be consuming more machine time because the periods have been artificially shortened. In addition, cyclic executives get complicated when they have to deal with aperiodic events. The rate monotonic approach, as will be discussed later, readily accommodates aperiodic processing. Finally, the rate monotonic utilization bound as computed by Theorem 2 is a function of the periods and computation times of the task set. The utilization bound can always be increased by transforming task periods, as described in the next section. 


\subsection{Stability Under Transient Overload}

In the previous section, the computation time of a task is assumed to be constant. However, in many applications, task execution times are often stochastic, and the worst-case execution time can be significantly larger than the average execution time. To have a reasonably high average processor utilization, we must deal with the problem of transient overload. We consider a scheduling algorithm to be stable if there exists a set of critical tasks such that all tasks in the set will meet their deadlines even if the processor is overloaded. This means that under worst-case conditions, tasks outside the critical set may miss their deadlines. The rate monotonic algorithm is stable in the sense that the set of tasks that never miss their deadlines does not change as the processor gets more overloaded or as task phasings change. Of course, which tasks are in the critical task set depends on the worst-case utilizations of the particular tasks being considered. The important point is that the rate monotonic theory guarantees that if such a set exists, it always consists of tasks with the highest priorities. This means that if a transient overload should develop, tasks with longer periods will miss their deadlines.

Of course, a task with a longer period could be more critical to an application than a task with a shorter perioc. One might attempt to ensure that the critical task always meets its deadline by assigning priorities according to a task's importance. However, this approach can lead to poor schedulability. That is, with this approach, deadlines of critical tasks might be met only when the total utilization is low.

The period transformation technique can be used to ensure high utilization while meeting the deadline of an important, long-period task. Period transformation means turning a longperiod important task into a high priority task by splitting its work over several short periods. For example, suppose task $\tau$ with a long period $T$ is not in the critical task set and must never miss its deadline. We can make $\tau$ simulate a short period task by giving it a period of $T / 2$ and suspending it after it executes half its worst-case execution time, $C / 2$. The task is then resumed and finishes its work in the next execution period. It still completes its total computation before the end of period $T$. From the viewpoint of the rate monotonic theory, the transformed task has the same utilization but a shorter period, $T / 2$, and its priority is raised accordingly. It is important to note that the most important task need not have the shortest period. We only need to make sure that it is among the first $n$ high priority tasks whose worst-case utilization is within the scheduling bound. A systematic procedure for period transformation with minimal task partitioning can be found in [7].

Period transformation allows important tasks to have higher priority while keeping priority assignments consistent with rate monotonic rules. This kind of transformation should be familiar to users of cyclic executives. The difference here is that we don't need to adjust the code segment sizes so different code segments fit into shared time slots. Instead, $\tau$ simply requests suspension after performing $C / 2$ amount of work. Alternatively, the runtime scheduler can be instructed to suspend the task after a certain amount of computation has 
been done, without affecting the application code. ${ }^{4}$

The period transformation approach has another benefit - it can raise the rate monotonic utilization bound. Suppose the rate monotonic utilization bound is $U_{\max }<100 \%$, i.e., total task utilization cannot be increased above $U_{\max }$ without missing a deadline. When a period transformation is applied to the task set, $U_{\max }$ will rise. For example:

Example 4: Let

- Task $\tau_{1}: C_{1}=4 ; T_{1}=10 ; U_{1}=.400$

- Task $\tau_{2}: C_{2}=6 ; T_{2}=14 ; U_{2}=.428$

The total utilization is .828 , which just equals the bound of Theorem 2 , so this set of two tasks is schedulable. If we apply Theorem 2 , we find:

$\begin{array}{lll}C_{1}+C_{2} \leq T_{1} & 4+6=10 & l=1, k=1 \\ \text { or } \quad 2 C_{1}+C_{2} \leq T_{2} & 8+6=14 & l=1, k=2\end{array}$

So Theorem 2 says the task set is just schedulable. Now suppose we perform a period transformation on task $\tau_{1}$, so $C_{1}^{\prime}=2$ and $T_{1}^{\prime}=5$. The total utilization is the same and the set is still schedulable, but when we apply Theorem 2 we find:

$\begin{array}{lll}C_{1}+C_{2} \leq T_{1} & 2+6>5 & l=1, k=1 \\ \text { or } 2 C_{1}+C_{2} \leq 2 T_{1} & 4+6=10 & l=2, k=1 \\ \text { or } 3 C_{1}+C_{2}<T_{2} & 6+6<14 & l=1, k=2\end{array}$

The third equation shows that the compute times for tasks $\tau_{1}$ and/or $\tau_{2}$ can be increased without violating the constraint. For example, the compute time of task $\tau_{1}$ can be increased by $2 / 3$ units to 2.667 , giving an uverall schedulable utilization of $2.667 / 5+6 / 14=.961$, or the compute time of Task $\tau_{2}$ can be increased to 8 , giving an overall schedulable utilization of $2 / 5+8 / 14=.971$. So the effect of the period transformation has been to raise the utilization bound from .828 to at least .961 and at most .971 . Indeed, if periods are uniformly harmonic, i.e., if each period is an integral multiple of each shorter period, the utilization bound of the rate monotonic algorithm is $100 \% .^{5}$ So the utilization bound produced by the rate monotonic approach is only an upper bound on what can be achieved if the periods are not transformed. Of course, as the periods get shorter, the scheduling overhead utilization in-

\footnotetext{
4The scheduler must ensure that $\tau$ is not suspended while in a critical region since such a suspension can cause other tasks to miss their deadlines. H the suspension time arrives but the task is in a critical region, then the suspension should be delayed until the task exits the critical region. To account for this effect on the

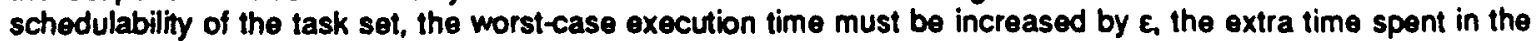
critical region, i.e., $t$ 's utilization becomes $(0.5 C+\varepsilon) 10.5 T$.
}

5For example, by transforming the periods in Example $380 \tau_{1}^{\prime}$ and $\tau_{2}^{\prime}$ both have periods of 50, the utilization bound is $100 \%$, i.e., $4.7 \%$ more work can be done without missing a deadline. 
creases, so the amount of useful work that can be done decreases. For example, before a period transformation, the utilization for a task, including scheduling overhead, is $(C+2 S) / T$. After splitting the period into two parts, the utilization is $(.5 C+25) / .5 T$, so scheduling overhead is a larger part of the total utilization. However, the utilization bound is also increased in general. If the increase in utilization caused by the scheduling overhead is less than the increase in the utilization bound, then the period transformation is a win - more useful work can be done while meeting all deadlines.

\subsection{Scheduling Both Aperiodic and Periodic Tasks}

It is important to meet the regular deadlines of periodic tasks and the response time requirements of aperiodic events. ("Aperiodic tasks" are used to service such events.) Let us begin with a simple example.

Example 5: Suppose that we have two tasks. Let $\tau_{1}$ be a periodic task with period 100 and execution time 99. Let $\tau_{2}$ be an aperiodic task that appears once within a period of 100 but the arrival time is random. The execution time of task $\tau_{2}$ is one unit. If we let the aperiodic task wait for the periodic task, then the average response time is about 50 units. The same can be said for a polling server, which provides one unit of service time in a period of 100 . On the other hand, we can deposit one unit of service time in a "ticket box" every 100 units of time; when a new "ticket" is deposited, the unused old tickets, if any, are discarded. With this approach, no matter when the aperiodic event arrives during a period of 100 , it will find there is a ticket for one unit of execution time at the ticket-box. That is, $\tau_{2}$ can use the ticket to preempt $\tau_{1}$ and execute immediately when the event occurs. In this case, $\tau_{2}$ 's response time is precisely one unit and the deadlines of $\tau_{1}$ are still guaranteed. This is the idea behind the deferrable server algorithm [4], which reduces aperiodic response time by a factor of about 50 in this example.

In reality, there can be many periodic tasks whose periods can be arbitrary. Furthermore, aperiodic arrivals can be very bursty, as for a Poisson process. However, the idea remains unchanged. We should allow the aperiodic tasks to preempt the periodic tasks subject to not causing their deadlines to be missed. It was shown in [4] that the deadlines of periodic tasks can be guaranteed provided that during a period of $T_{a}$ units of time, there are no more than $\mathrm{C}_{\mathrm{a}}$ units of time in which aperiodic tasks preempt periodic tasks. In addition, the total periodic and aperiodic utilization must be kept below $\left(U_{a}+\ln \left[\left(2+U_{a}\right) /\left(2 U_{a}+1\right)\right)\right]$, where $U_{a}=C_{a} T_{a}$. And the server's period must observe the inequality " $T_{a} \leq\left(T-C_{a}\right)^{*}$, where $\mathrm{T}$ is the period of a periodic task whose priority is next to the server.

Compared with background service, the deferrable server algorithm typically improves aperiodic response time by a factor between 2 and 10 [4]. Under the deferrable server algorithm, both periodic and aperiodic task modules can be modified at will as long as the utilization bound is observed. Figure 2-1 illustrates the relative performance between background execution, the deferrable server algorithm, and polling. The workload is $60 \%$ periodic and $20 \%$ aperiodic. We assume a Polsson arrival process and exponentially distri- 
buted execution time for the aperiodic tasks. Since the mean aperiodic workload is fixed at $20 \%$, short mean interarrival times imply short mean execution times. As we can see from Figure 2-1, the deferrable server is most effective for frequent arrivals with small service times.

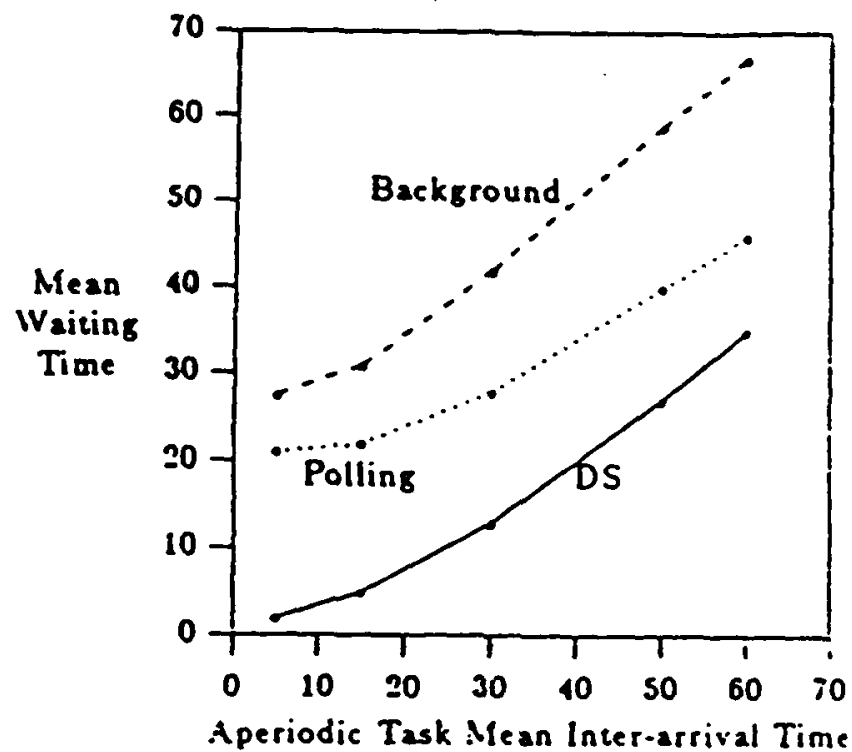

Figure 2-1: Scheduling Both Aperiodic and Periodic Tasks

A variation to the deferrable server algorithm is known as the sporadic server algorithm [9]. As for the deferrable server algorithm, we allocate $C_{a}$ units of computation time within a period of $T_{a}$ units of time. However, the $\mathrm{C}_{\mathrm{a}}$ of the server's budget is not refreshed until the budget is consumed. ${ }^{6}$ From a capacity planning point of view, a sporadic server is equivalent to a periodic task that performs polling. That is, we can place sporadic servers at various priority levels and use only Theorems 1 and 2 to perform a schedulability analysis. Sporadic and deferrable servers have similar performance gains over polling, because any time an aperiodic task arrives, it can use the allocated budget immediately. When polling is used, however, an aperiodic arrival generally needs to wait for the next instant of polling. The sporadic server has the least runtime overhead. Both the polling and the deferrable servers have to be serviced periodically, even if there are no aperiodic arrivals. ${ }^{7}$ There is no overhead for the sporadic server until its execution budget has been consumed. In particular, there is no overhead if there are no aperiodic arrivals. Therefore, the sporadic server is especially ruitable for handling emergency aperiodic events that occur rarely but must be responded t quickly.

\footnotetext{
Earty refreahing is also posstble under certain conditions. See [9].

7The ticket box must be refreshed at the end of each deferrable server's period.
} 


\subsection{Task Synchronization}

In the previous sections we have discussed the scheduling of independent tasks. Tasks, however, do interact. In this section, we will discuss how the rate monotonic scheduling theory can be applied to real-time tasks that must interact. The discussion is limited in this paper to scheduling within a uniprocessor. Readers who are interested in the multiprocessor synchronization problem should see [6].

Common synchronization primitives include semaphores, locks, monitors, and Ada rendezvous. Although the use of these or equivalent methods is necessary to protect the consistency of shared data or to guarantee the proper use of non-preemptable resources, their use may jeopardize the ability of the system to meet its timing requirements. In fact, a direct application of these synchronization mechanisms may lead to an indefinite period of priority inversion and low schedulability.

Example 6: Suppose $J_{1}, J_{2}$, and $J_{3}$ are three jobs arranged in descending order of priority with $J_{1}$ having the highest priority. We assume that jobs $J_{1}$ and $J_{3}$ share a data structure guarded by a binary semaphore $S$. Suppose that at time $t_{1}$, job $J_{3}$ locks the semaphore $S$ and executes its critical section. During the execution of the critical section of job $J_{3}$, the high priority job $J_{1}$ is initiated, preempts $J_{3}$ and later attempts to use the shared data. However, job $J_{1}$ will be blocked on the semaphore $S$. We would hope that $J_{1}$, being the highest priority job, is blocked no longer than the time for job $J_{3}$ to complete its critical section. However, the duration of blocking is, in fact, unpredictable. This is because job $J_{3}$ can be preempted by the intermediate priority job $J_{2}$. The blocking of $J_{3}$, and hence that of $J_{1}$, will continue until $J_{2}$ and any other pending intermediate jobs are completed.

The blocking period in this example can be arbitrarily long. This situation can be partially remedied if a job in its critical section is not allowed to be preempted; however, this solution is only appropriate for very short critical sections because it creates unnecessary blocking. For instance, once a low priority job enters a long critical section, a high priority job that does not access the shared data structure may be needlessly blocked.

The priority ceiling protocol is a real-time synchronization protocol with two important properties: 1) freedom from mutual deadlock, and 2) bounded blocking, i.e., at most one lower priority task can block a higher priority task $[1,8]$. There are two ideas in the design of this protocol. First is the concept of priority inheritance: when a task $\tau$ blocks the execution of higher priority tasks, task $\tau$ executes at the highest priority level of all the tasks blocked by $\tau$. Secondly, we must guarantee that a critical section is allowed to start execution only if the section will always execute at a priority level that is higher than the (inherited) priority levels of any preempted critical sections. It was shown in [8] that such a prioritized total ordering in the execution of critical sections leads to the two desired properties. To achieve such prioritized total ordering, we define the priority ceiling of a binary semaphore $S$ to be the highest priority of all tasks that may lock $S$. When a task $\tau$ attempts to execute one of its critical sections, it will be suspended unless its priority is higher than the priority ceilings of all semaphores currently locked by tasks other than $\tau$. If task $\tau$ is unable to enter its critical 
section for this reason, the task that holds the lock on the semaphore with the highest priority ceiling is said to be blocking $\tau$ and hence inherits the priority of $\tau$. As long as a task $\tau$ is not attempting to enter one of its critical sections, it will preempt every task that has a lower priority.

Example 7: Suppose that we have two jobs $J_{1}$ and $J_{2}$ in the system. In addition, there are two shared data structures protected by binary semaphores $S_{1}$ and $S_{2}$ respectively. Suppose the sequence of processing steps for each job is as follows.

$$
\begin{aligned}
& J_{1}=\left\{\cdots, P\left(S_{1}\right), \cdots, P\left(S_{2}\right), \cdots, V\left(S_{2}\right), \cdots, V\left(s_{1}\right), \cdots\right\} \\
& J_{2}=\left\{\cdots, P\left(s_{2}\right), \cdots, P\left(S_{1}\right), \cdots, V\left(S_{1}\right), \cdots, V\left(s_{2}\right), \cdots\right\}
\end{aligned}
$$

Recall that the priority of job $J_{1}$ is assumed to be higher than that of job $J_{2}$. Thus, the priority ceilings of both semaphores $S_{1}$ and $S_{2}$ are equal to the priority of job $J_{1}$. Suppose that at time $t_{0}, J_{2}$ is initiated and it begins execution and then locks semaphore $S_{2}$. At time $t_{1}$, job $J_{1}$ is initiated and preempts job $J_{2}$ and at time $t_{2}$, job $J_{1}$ tries to enter its critical section by making an indivisible system call to execute $P\left(S_{1}\right)$. However, the runtime system will find that the priority of $J_{1}$ is not higher than the priority ceiling of locked semaphore $S_{2}$. Hence, the runtime system suspends job $J_{1}$ without locking $S_{1}$. Job $J_{2}$ now inherits the priority of job $J_{1}$ and resumes execution. Note that $J_{1}$ is blocked outside its critical section. As $J_{1}$ is not given the lock on $S_{1}$ but suspended instead, the potential deadlock involving $J_{1}$ and $J_{2}$ is prevented. Once $J_{2}$ exits its critical section, it will return to its assigned priority and immediately be preempted by job $J_{1}$. From this point on, $J_{1}$ will execute to completion, and then $J_{2}$ will resume its execution until its completion.

Let $B_{i}$ be the longest duration of blocking that can be experienced by task $\tau_{i}$. The following two theorems indicate whether the deadlines of a set of periodic tasks can be met if the priority ceiling protocol is used.

Theorem 3: A set of $n$ periodic tasks using the priority ceiling protocol can be scheduled by the rate monotonic algorithm, for all task phasings, if the following condition is satisfied [8]:

$$
\frac{C_{1}}{T_{1}}+\cdots+\frac{C_{n}}{T_{n}}+\max \left(\frac{B_{1}}{T_{1}}, \cdots, \frac{B_{n-1}}{T_{n-1}}\right) \leq n\left(2^{1 / n-1}\right)
$$

Theorem 4: A set of $n$ periodic tasks using the priority ceiling protocol can be scheduled by the rate monotonic algorithm for all task phasings if the following condition is satisfied [8].

$$
\forall i, 1 \leq i \leq n, \quad \min _{(k, l) \in R_{i}}\left(\sum_{j=1}^{i-1} C_{j} \frac{1}{l T_{k}}\left\lceil\frac{l T_{k}}{T_{j}}\right]+\frac{C_{i}}{l T_{k}}+\frac{B_{i}}{l T_{k}}\right) \leq 1
$$

where $C_{i}, T_{i}$, and $R_{i}$ are defined in Theorem 2, and $B_{i}$ is the worst-case blocking time for $\tau_{i}$. 
Remark: Theorems 3 and 4 generalize Theorems 1 and 2 by taking blocking into consideration. The $B_{i}$ 's in Theorems 3 and 4 can be used to account for any delay caused by resource sharing. Note that the upper limit of the summation in the theorem is $(i-1)$ instead of $i$, as in Theorem 2.

In the application of Theorems 3 and 4, it is important to realize that under the priority ceiling protocol, a task $\tau$ can be blocked by a lower priority task $\tau_{L}$ if $\tau_{L}$ may lock a semaphore $S$ whose priority ceiling is higher than or equal to the priority of task $\tau$, even if $\tau$ and $\tau_{L}$ do not share any semaphore. For example, suppose that $\tau_{L}$ locks $S$ first. Next, $\tau$ is initiated and preempts $\tau_{L}$. Later, a high priority task $\tau_{H}$ is initiated and attempts to lock $S$. Task $\tau_{H}$ will be blocked. Task $\tau_{L}$ now inherits the priority of $\tau_{H}$ and executes. Note that $\tau$ has to wait for the critical section of $\tau_{L}$ even though $\tau$ and $\tau_{L}$ do not share any semaphore. We call such blocking push-through blocking. Push-through blocking is the price for avoiding unbounded priority inversion. If task $\tau_{L}$ does not inherit the priority of $\tau_{H}$, task $\tau_{H}$ can be indirectly preempted by task $\tau$ and all the tasks that have priority higher than that of $\tau_{L}$. Finally, we want to point out that even if task $\tau_{H}$ does not attempt to lock $S$ but attempts to lock another unlocked semaphore, $\tau_{H}$ will still be blocked by the priority ceiling protocol because the priority of $\tau_{H}$ is not higher than the priority ceiling of $S$. We term this form of blocking as ceiling blocking. Ceiling block is the price for ensuring the freedom of deadlock and the property of a task being blocked at most once.

\subsection{An Example Application of the Theory}

In this section, we give a simple example to illustrate the application of the scheduling theory.

Example 8: Consider the following task set.

1. Emergency handling task: execution time $=5 \mathrm{msec}$; worst case interarrival time = 50 msec; deadline is 6 msec after arrival.

2. Aperiodic event handling tasks: average execution time $=2 \mathrm{msec}$; average inter-arrival time $=\mathbf{4 0} \mathrm{msec}$; fast response time is desirable but there are no hard deadlines.

3. Periodic task $\tau_{1}$ : execution time $=20 \mathrm{msec}$; period $=100 \mathrm{msec}$; deadline is at the end of each period.

In addition, $\tau_{3}$ may block $\tau_{1}$ for 10 msec by using a shared communication server, and task $\tau_{2}$ may block $\tau_{1}$ for 20 msec by using a shared data object.

4. Periodic task $\tau_{2}$ : execution time $=40 \mathrm{msec}$; period $=150 \mathrm{msec}$; deadline is 20 msec before the end of each period.

5. Periodic task $\tau_{3}$ : execution time $=100 \mathrm{msec}$; period $=350 \mathrm{msec}$; deadline is at the end of each period.

Solution: First, we create a sporadic server for the emergency task, with a period of 50 msec and a service time 5 msec. Since the server has the shortest period, the rate monotonic algorithm will give this server the highest priority. It follows that the emergency task can meet its deadline. 
Since the aperiodic tasks have no deadlines, they can be assigned a low background priority. However, since fast response time is desirable, we create a sporadic server executing at the second highest priority. The size of the server is a design issue. A larger server (i.e., a server with higher utilization) needs more processor cycles but will give better response time. In this example, we choose a large server with a period of $100 \mathrm{msec}$ and a service time of 10 msec. We now have two tasks with a period of $100 \mathrm{msec}$, the aperiodic server and periodic task $\tau_{1}$. The rate monotonic algorithm allows us to break the tie arbitrarily, and we let the server have the higher priority.

We now have to check if the three periodic tasks can meet their deadlines. Since under the priority ceiling protocol a task can be blocked by lower priority tasks at most once, the maximal blocking time for task $\tau_{1}$ is $B_{1}=\max (10,20) \mathrm{msec}=20 \mathrm{msec}$. Since $\tau_{3}$ may lock the semaphore $S_{c}$ associated with the communication server and the priority ceiling of $S_{c}$ is higher than that of task $\tau_{2}$, task $\tau_{2}$ can be blocked by task $\tau_{3}$ for $10 \mathrm{msec}{ }^{8}$ Finally, task $\tau_{2}$ has to finish 20 msec earlier than the nominal deadline for a periodic task. This is equivalent to saying that $\tau_{2}$ will always be blocked for an additional $20 \mathrm{msec}$ but its deadline is at the end of the period. Hence, $B_{2}=(10+20) \mathrm{msec}=30 \mathrm{msec}{ }^{9}$ At this point, we can directly apply Theorem 4. However, we can also reduce the number of steps in the analysis by noting that period 50 and 100 are harmonics and we can treat the emergency server as if it has a period of $100 \mathrm{msec}$ and a service time of $10 \mathrm{msec}$, instead of a period of $50 \mathrm{msec}$ and a service time of $5 \mathrm{msec}$. We now have three tasks with a period of $100 \mathrm{msec}$ and an execution time of $20 \mathrm{msec}, 10 \mathrm{msec}$, and $10 \mathrm{msec}$ respectively. For the purpose of analysis, these three tasks can be replaced by a single periodic task with a period of $100 \mathrm{msec}$ and an execution time of $40 \mathrm{msec}(20+10+10)$. We now have the following three equivalent periodic tasks for analysis:

- Task $\tau_{1}: C_{1}=40 ; T_{1}=100 ; B_{1}=20 ; U_{1}=0.4$

- Task $\tau_{2}: C_{2}=40 ; T_{2}=150 ; B_{2}=30 ; U_{2}=0.267$

- Task $\tau_{3}: C_{3}=100 ; T_{3}=350 ; B_{3}=0 ; U_{3}=0.286$

Using Theorem 4:

1. Task $\tau_{1}$ : Check $C_{1}+B_{1} \leq T_{1}$. Since $40+20 \leq 100$, task $\tau_{1}$ is schedulable.

2. Task $\tau_{2}$ : Check whether either

$$
\begin{array}{lll}
C_{1}+C_{2}+B_{2} \leq T_{1} & 40+40+30>100 \\
\text { or } & 2 C_{1}+C_{2}+B_{2} \leq T_{2} & 80+40+30=150
\end{array}
$$

Task $\tau_{2}$ is schedulable and in the worst-case phasing will meet its deadine exactly at time 150 .

\footnotetext{
This may occur if $\tau_{3}$ blocks $\tau_{1}$ and inherits the priority of $\tau_{1}$.

Wote that the blocked-at-most-once result does not apply here. It only applies to blocking caused by task synchronization using the priority coiling frotocol.
} 
3. Task $\tau_{3}$ : Check whether either

$$
\begin{array}{lll} 
& C_{1}+C_{2}+C_{3} \leq T_{1} & 40+40+100>100 \\
\text { or } & 2 C_{1}+C_{2}+C_{3} \leq T_{2} & 80+40+100>150 \\
\text { or } & 2 C_{1}+2 C_{2}+C_{3} \leq 2 T_{1} & 80+80+100>200 \\
\text { or } & 3 C_{1}+2 C_{2}+C_{3} \leq 2 T_{2} & 120+80+100=300 \\
\text { or } & 4 C_{1}+3 C_{2}+C_{3} \leq T_{3} & 160+120+100>350
\end{array}
$$

Task $\tau_{3}$ is also schedulable and in the worst-case phasing will meet its deadline exactly at time 300 . It follows that all three periodic tasks can meet their deadlines.

We now determine the response time of the aperiodics. The server capacity is $10 \%$ and the average aperiodic workload is $5 \%(2 / 40)$. Because most of the aperiodic arrivals can find "tickets," we would expect a good response time. Indeed, using a M/N/12] approximation for the lightly loaded server, the expected response time for the aperiodics is $W=E[S] / 1-$ $p)=2 /(1-(0.05 / 0.10))=4 \mathrm{msec}$, where $E[S]$ is the average execution time of aperiodic tasks and $\rho$ is the average server utilization. Finally, we want to point out that although the worst-case total periodic and server workload is $95 \%$, we can still do quite a bit of background processing since the soft deadline aperiodics and the emergency task are unlikely to fully utilize the servers. 


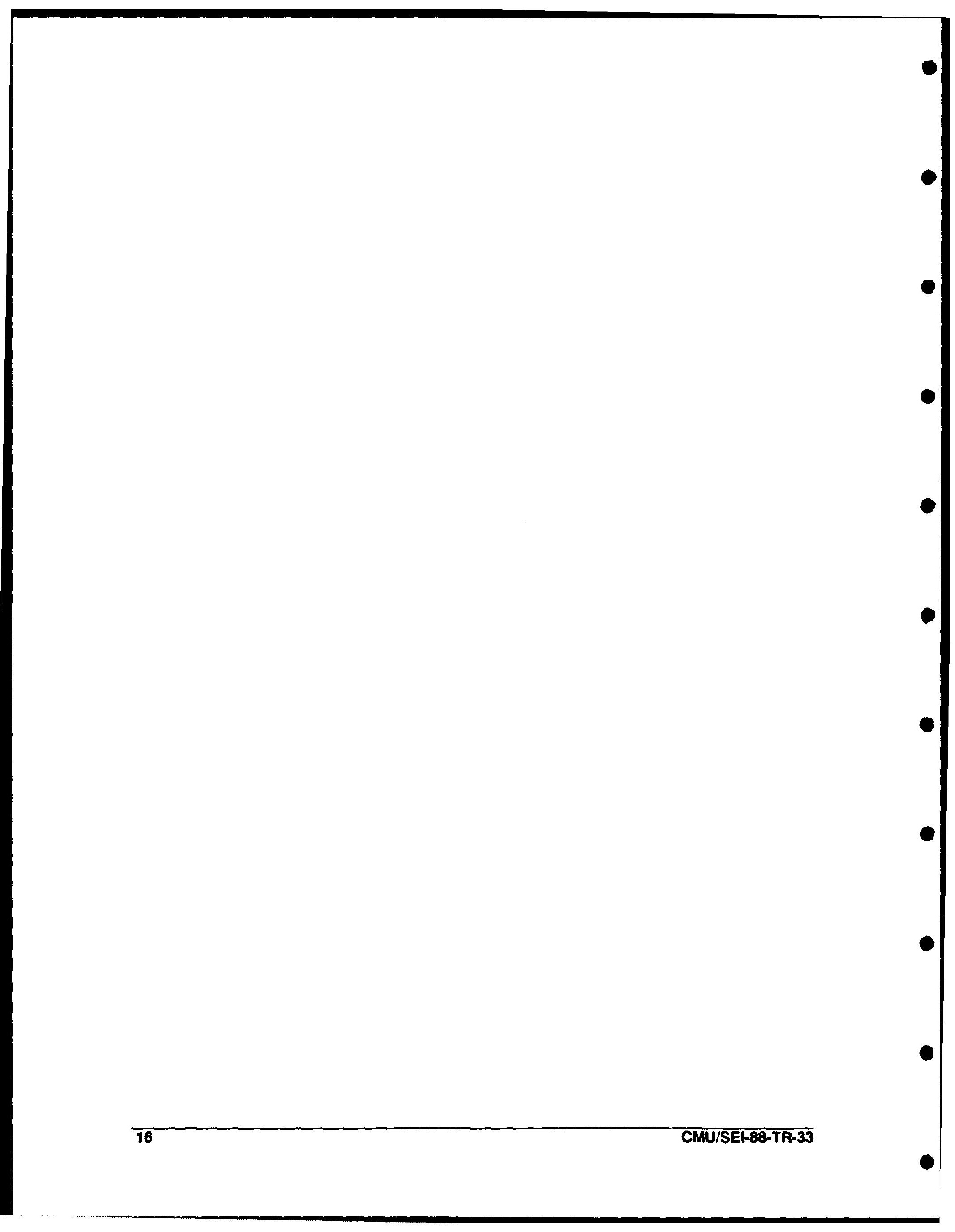




\section{Real-Time Scheduling in Ada}

The Real-Time Scheduling in Ada Project at the Software Engineering Institute is a cooperative effort between the SEI, system developers in industry, Ada vendors, and DoD agencies. It aims at applying the scheduling theory reviewed in Section 2 to the design and implementation of hard real-time systems in Ada. The deferred server, sporadic server, and priority ceiling scheduling algorithms have all been developed at Carnegie Mellon University. We call these analytic scheduling algorithms because the overall timing behavior of a system is subject to mathematical analysis, starting with the basic rate monotonic theorems. The project is implementing these analytic scheduling algorithms using an Ada runtime system, and is coding examples of real-time systems to evaluate the suitability of the whole approach.

While the use of Ada for real-time systems presents many practical, implementationdependent questions such as the cost of performing a rendezvous, there are other important questions about the suitability of the concepts and scheduling rules of Ada. For example, tasks in Ada run non-deterministically, making it hard for traditional real-time programmers to decide whether any tasks will meet their deadlines. In addition. the scheduling rules of Ada don't seem to support prioritized scheduling well. Prioritized tasks are queued in FIFO order rather than by priority, high priority tasks can be delayed indefinitely when calling low priority tasks, and task priorities cannot be changed when application demands change at runtime. Fortunately, it appears that none of these problems present insurmountable difficulties; solutions exist within the current language framework, although some language changes would be helpful. In the SEI Real-Time Scheduling in Ada Project, we are investigating how to apply analyzable scheduling algorithms within the Ada scheduling rules; we are also investigating how the scheduling rules of Ada might be changed to make Ada more directly suitable for real-time work. In addition, we are formulating Ada coding guidelines to use when coding hard real-time systems. These guidelines allow the project's scheduling algorithms to be applied and supported within the existing language rules. The guidelines are still evolving and being evaluated, but so far, it seems likely they will meet the needs of a useful range of systems.

\subsection{On Ada Scheduling Rules}

First of all, the Ada tasking model is well-suited, in principle, to the use of real-time scheduling algorithms. When using this approach, a programmer doesn't need to know when each task is running to be sure that deadlines will be met. That is, both Ada and the theory abstract away the details of an execution timeline and view tasks as the basic unit of abstraction for the management of concurrency. Although Ada tasks fit well with the theory at the conceptual level, Ada and the theory differ on the rules for determining when a task is eligible to run and its execution priority. For example, if a high priority task calls a lower priority task that is in rendezvous with another low priority task, the rendezvous continues at the priority of the task being served Instead of being increased because a high priority task is waiting. Under these circumstances, the high priority task can be blocked as long as there are medium priority jobs able to run. But there are a variety of solutions to this prob- 
lem. The most general solution within the constraints of the language is simply to not use pragma PRIORITY at all. If all tasks in a system have no assigned priority, then the scheduler is free to use any convenient algorithm for deciding which eligible task to run. An implementation-dependent pragma could be used to give "scheduling priorities" to tasks, i.e., indications of scheduling importance that would be used in accordance with analytic scheduling algorithms. This approach would even allow "priorities" to be changed dynamically by the programmer because such changes only affect the scheduling of tasks that, in a legalistic sense, have no Ada priorities at all. The only problem with this approach is that tasks are still queued in FIFO order rather than by priority. However, this problem can often be solved by using a coding style that prevents queues from having more than one task. making the FIFO issue irrelevant. Of course, telling programmers to assign "scheduling priorities" to tasks but not to use pragma PRIORITY, and being careful to avoid queueing tasks surely says we are fighting the language rather than taking advantage of it.

While this kind of drastic approach may be necessary in some cases, it nonetheless seems likely that many real-time systems can be programmed satisfactorily using the concept of priorities in Ada and giving an appropriate interpretation to Ada scheduling rules. The relevant Ada rules are:

- Task priorities: fixed. This rule is inappropriate when task priorities need to be changed at runtime. For example, when a new mode is initiated, the frequency of a task and/or its criticality may change, implying its priority must change. In addition, the priority of a sporadic server for aperiodic events needs to be lowered when "tickets" are used up, and needs to be raised when new "tickets" arrive.

Solution: Nhen an application needs to adjust the priority of a task at runtime, this task should be declared as having no Ada priority. The runtime system can then be given a way of adjusting the priority of the task.

- CPU allocation: priorities must be observed. Ada requires that the highest priority task eligible to run be given the CPU when this is "sensible." "Sensible" for a uniprocessor is usually interpreted to mean that if a call by an executing task can be accepted, the call must be accepted and no lower priority task can be allowed to execute. Although this interpretation may seem to be obviously the best, it is in fact not correct for the priority ceiling protocol, which gives better service to high priority tasks by ensuring that they are blocked at most once by lower priority tasks. For example (see Figure 3-1), suppose a critical region is implemented as a server task $S$, whose accept statements are the critical regions guarded by a semaphore, and suppose a low priority task is in rendezvous with $S$. Also suppose the priority ceiling of $S$ is $H$ (i.e., the highest priority task that can call $S$ has priority $H$ ). Now suppose execution of the rendezvous is preempted by a medium priority task $M$, whose priority is less than $H$. Suppose $M$ tries to call T, a server other than $S$. The priority ceiling protocol says that the call from $M$ must be blocked, but the normal interpretation of Ada's scheduling rules would imply that the call of $M$ must be accepted since $M$ is the highest priority task that is eligible to run and $T$ is able to accept the call.

Solution: $M$ can be suspended just as it is about to call $T$ because the priority ceiling protocol says it isn't "sensible" to let server T execute on behalf of M. So $M$ is suspe-ided, and the execution of $S$ continues to the end of the rendezvous. At that point, the priority ceiling protocol allows the call from $M$ to succeed. 
Alternatively, give $S$ and $T$ an undefined priority. That is, the Ada rules say $S$ has no defined priority outside a rendezvous and within a rendezvous; it has "at least" the priority of the calling task. These rules give an implementation sufficient freedom to support the priority ceiling protocol directly. For example, $S$ is allowed to preempt $M$ just at the point where $M$ is about to call $T$.

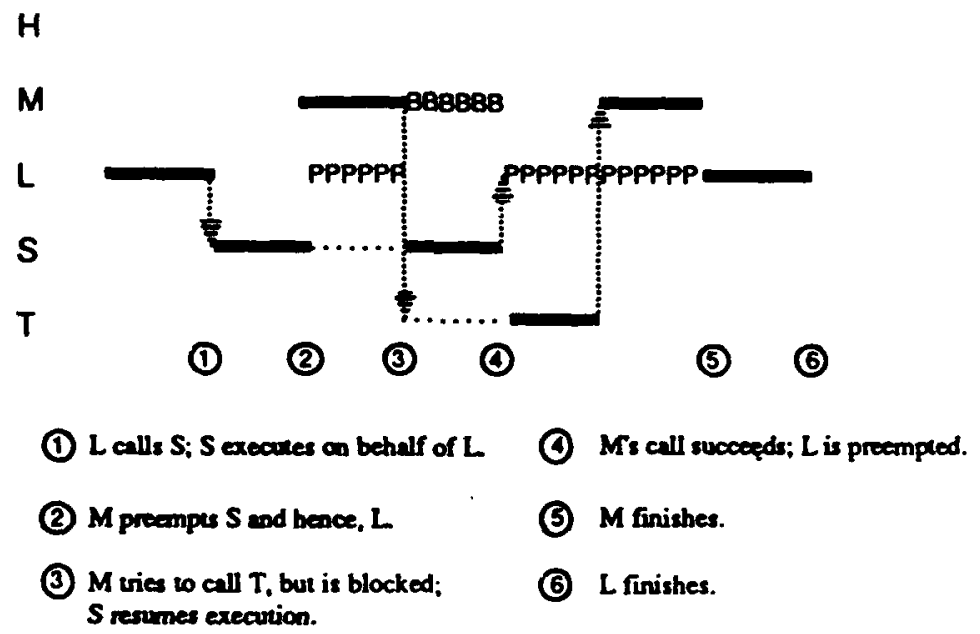

Flgure 3-1: Blocking Due to the Prionity Celiling Protocol

- Hardware task priority: always higher than software task priorities. This Ada rule reflects current hardware designs, but hardware intemupts should not always have the highest priority. from the viewpoint of the rate monotonic theory.

Solution: When handling an internupt that, in terms of the rate monotonic theory. should have a lower priority than the priority of some application task, keep the internupt handling actions short (which is already a common practice) and include the interrupt handling duration as blocking time in the rate monotonic analysis.

- Priority rules for task rendezvous:

- Selective walt: priority can be ignored. That is, the scheduler is allowed, but not required, to take priorities into account.

Solution: Ensure the runtime system takes priorities of waiting tasks into account.

- FIFO entry queues: the priority of calling tasks must be ignored.

Solution: Otten, it is possible to prevent queves from being formed (as explained in the next section). If there are no queues, no issue of FIFO queueing arises. If $t \mathrm{t}$ is impossible to prevent the formation of an entry queve, entry families can be used to get the effect of prioritized queueing.

- Called task priority: only increased during rendezvous. 
Solution: Don't specify the priority of the called task. If the called task has no assigned priority, its effective priority can be increased according to priority inheritance or other scheduling rules. Alternatively, give the called task a priority just higher than the priority of any of its callers. From an analytical point of view, this causes no additional blocking in the worst case, i.e., this approach does not reduce the schedulability of the system. However, when the server is called by a low priority task, it can now preempt some tasks that would otherwise be executable under the priority ceiling protocol. This can cause the average response time for aperiodic servers to be worse, and can increase the probability that a non-critical task will miss its deadline in a transient overload situation. But if the service time for low priority tasks is short, these negative effects will be negligible, so this is often a viable approach that can be used with today's Ada runtime systems.

From what our project has learned so far, it seems to be possible in practice to support analytic scheduling algorithms under current Ada rules by using a combination of runtime system modifications and appropriate coding protocols. Of course, it would be better if the language did not get in the way of priority scheduling principles. The future revision of Ada should probably revise some of these rules so priority-based scheduling can be supported more directly within the language.

\subsection{Ada Real-Time Design Guidelines}

While different guidelines can be applied to different real-time programming problems, the guidelines developed in the SEI project reflect a basic principle of real-time programming write systems in a way that minimizes the duration of priority inversion. In other words, minimize the time a high priority task has to wait for the execution of lower priority tasks.

For example, consider a set of periodic tasks that must exchange data among themselves or that must call a communication server. The periodic tasks are called client tasks. They do not call each other. Whenever they must read or write shared data or send a message, they call a server task whose entries represent the critical regions. The client tasks are given priorities according to rate monotonic principles, i.e., tasks with the shortest periods are given the highest priorities. There are two options when assigning a priority to a server. If the Ada runtime system supports the priority ceiling protocol directly, then give the server a low priority or an undefined priority. In addition, tell the runtime system the priority ceiling of the server, l.e., the highest priority of all its clients. The server will then, in effect, execute at its client's priority when there are no other callers. When a high priority client wants to call a server, the call may be blocked in accordance with priority ceiling rules because these rules specify when it is "sensible" to allow the high priority task to continue to execute.

If the priority ceiling protocol is not supported directly by the runtime system, the priority ceiling protocol can be approximated by assigning each server task a unique priority that is one greater than the priority of its highest priority caller. This approach can be used today with all Ada implementations. Theoretically, assigning the server the same priority as its 
highest priority caller is sufficient for the approximation. However, different Ada implementations treat tasks of equal priority differently. We have found that some implementations are inconsistent with the treatment demanded by the priority ceiling protocol for equal priority tasks, namely, they do not give preference to the server. Increasing the priority by one avoids this probiem as long as there is no client task at this priority level.

With either approach, as long as a server need not synchronize with external events, it will be either waiting for a client or serving its first caller at a priority that prevents other clients from calling the server. As a result, the server task never has more than one client on its queues, so prioritized queueing is not a problem. However, when a server needs to synchronize with external $\mathrm{VO}$ events, it is suspended while in a rendezvous. Other client tasks can then execute and attempt to call the server, and FIFO queues can develop. To ensure that entry queues do not develop, the runtime system should suspend tasks that attempt to call a server and allow some lower priority task to run. This has the same effect as a prioritized queue because the suspended tasks will be awakened in priority order; the highest priority calling task will have its call accepted first. If this solution is not supported by the runtime system, then entry families must be used to simulate priority queues.

From a theoretical viewpoint, the time spent in each rendezvous with a server is counted as part of the computing time, $C_{i}$, for the client task $\tau_{j}$. Since the use of servers is a synchronization problem, Theorems 3 and 4 apply. Under the priority ceiling protocol, the maximal blocking time for a client task at priority level $i$ is the longest entry call by a lower level client task to a server whose priority ceiling is equal to or higher than the priority of $i$. The worst-case blocking time is the same whether the ceiling protocol is implemented directly or is approximated by giving the server an appropriately high priority. The essential difference between the direct implementation and the approximation method is that for the direct implementation, the server priority will be raised only when necessary. This tends to generate less blocking on average and hence better response time when aperiodic tasks have a priority below that of a server's ceiling; in addition, in transient overload situations, non-critical periodic tasks having a priority lower than a server's ceiling are less likely to miss their deadlines. In short, the direct implementation gives better average case behavior, especially when the entry calls are relatively long. When the entry calls are short compared with client task execution times, the performance difference is insignificant. 


\section{Conclusion}

Ada tasking was intended to be used for real-time programming. However, the Ada tasking model represents a fundamental departure from the traditional cyclical executive model. Indeed, the dynamic preemption of tasks at runtime generates non-deterministic timelines that are at odds with the very idea of a fixed execution timeline required by a cyclical executive.

In this paper, we have reviewed some important results of priority scheduling theory. Together with Ada tasking, they allow programmers to reason with confidence about timing correctness at the tasking level of abstraction. As long as the analytic scheduling algorithms are supported by the runtime system and the resource utilization bounds on CPU, 1/O drivers, and communication media are observed, the timing constraints will be guaranteed. Even if there is a transient overload, the tasks missing deadlines will be in a predefined order.

Although the treatment of priorities by the current Ada tasking model can and should be improved, most of the scheduling algorithms can be used today within the existing Ada rules if an appropriate coding and design approach is taken, and if schedulers are written to take full advantage of certain coding styles and the existing flexibility in the scheduling rules. Additional reports on how this can be done are in preparation at the Software Engineering Institute. 


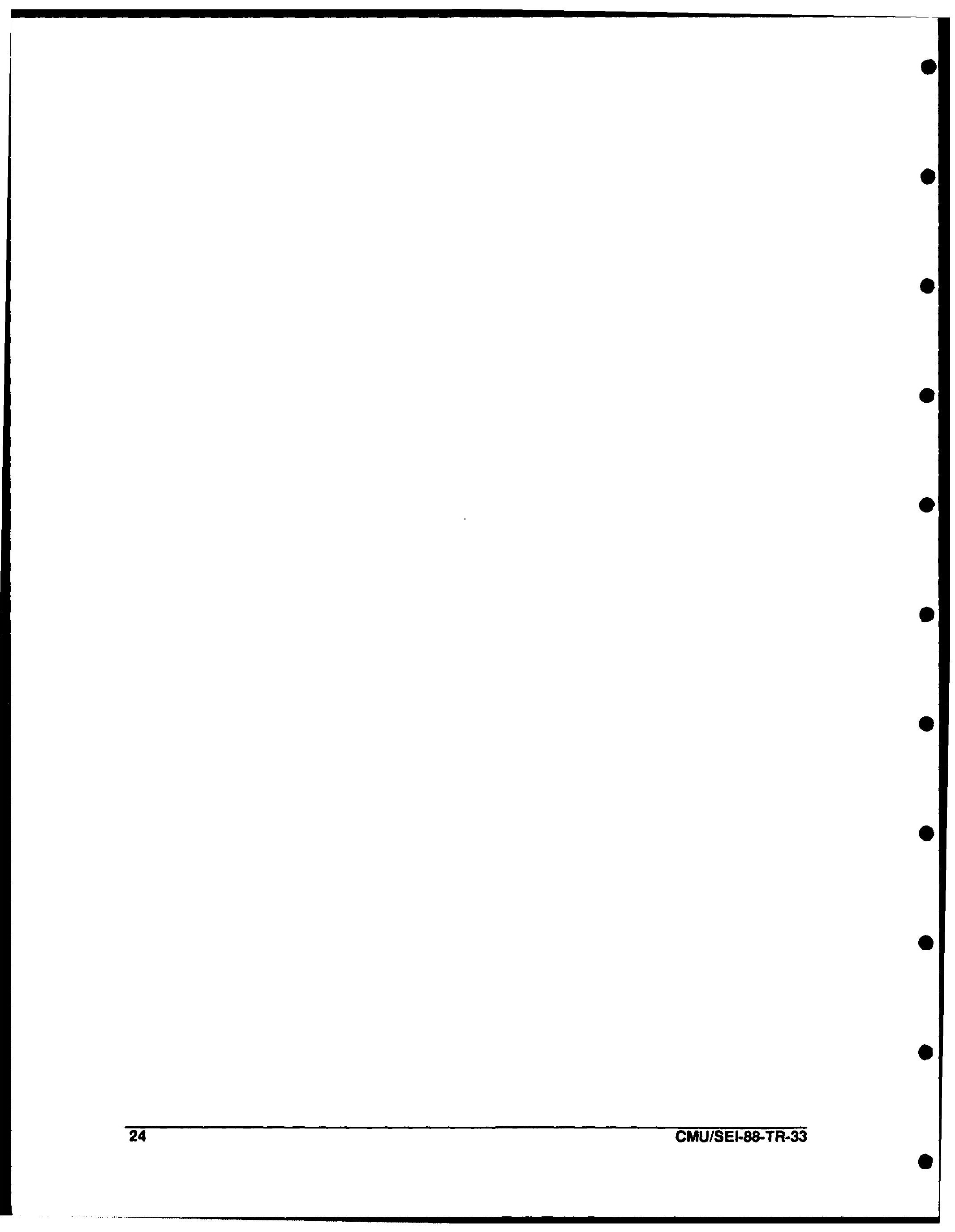




\section{References}

[1] Goodenough, J. B., and Sha, L.

The Priority Ceiling Protocol: A Method for Minimizing the Blocking of High Priority Ada Tasks.

To appear in the Proceedings of the 2nd ACM International Workshop on Real-Time Ada lssues, 1988.

[2] Kleinrock, L.

Queueing Systems, Vol I.

John Wiley \& Sons, 1975.

[3] Lehoczky, J. P., Sha, L. and Ding, Y.

The Rate Monotonic Scheduling Algorithm-Characterization and Average Case Behavior.

Technical Report, Department of Statistics, Carnegie Mellon University, 1987.

[4] Lehoczky, J. P., Sha L. and Strosnider, J.

Enhancing Aperiodic Responsiveness in A Hard Real-Time Environment.

IEEE Real-Time System Symposium, 1987.

[5] Liu, C. L. and Layland J. W.

Scheduling Algorithms for Multiprogramming in a Hard Real Time Environment.

JACM 20 (1):46 - 61, 1973.

[6] Rajkumar, R., Sha, L., and Lehockzy J.P.

Real-Time Synchronization Protocols for Multiprocessors.

To appear in Proceedings of the IEEE Real-Time Systems Symposium , 1988.

[7] Sha, L., Lehoczky, J. P. and Rajkumar, R.

Solutions for Some Practical Problems in Prioritized Preemptive Scheduling. IEEE Real-Time Systems Symposium , 1986.

[8] Sha, L., Rajkumar, R. and Lehoczky, J. P.

Priority Inheritance Protocols: An Approach to Real-Time Synchronization.

To appear in IEEE Transactions on Computers, 1987.

[9] Sprunt, B., Sha, L. and Lehoczky, J. P.

Scheduling Sporadic and Aperiodic Events in a Hard Real-Time System.

Technical Report (in preparation), 1988. 


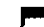


REPORT DOCUMENTATION PAGE

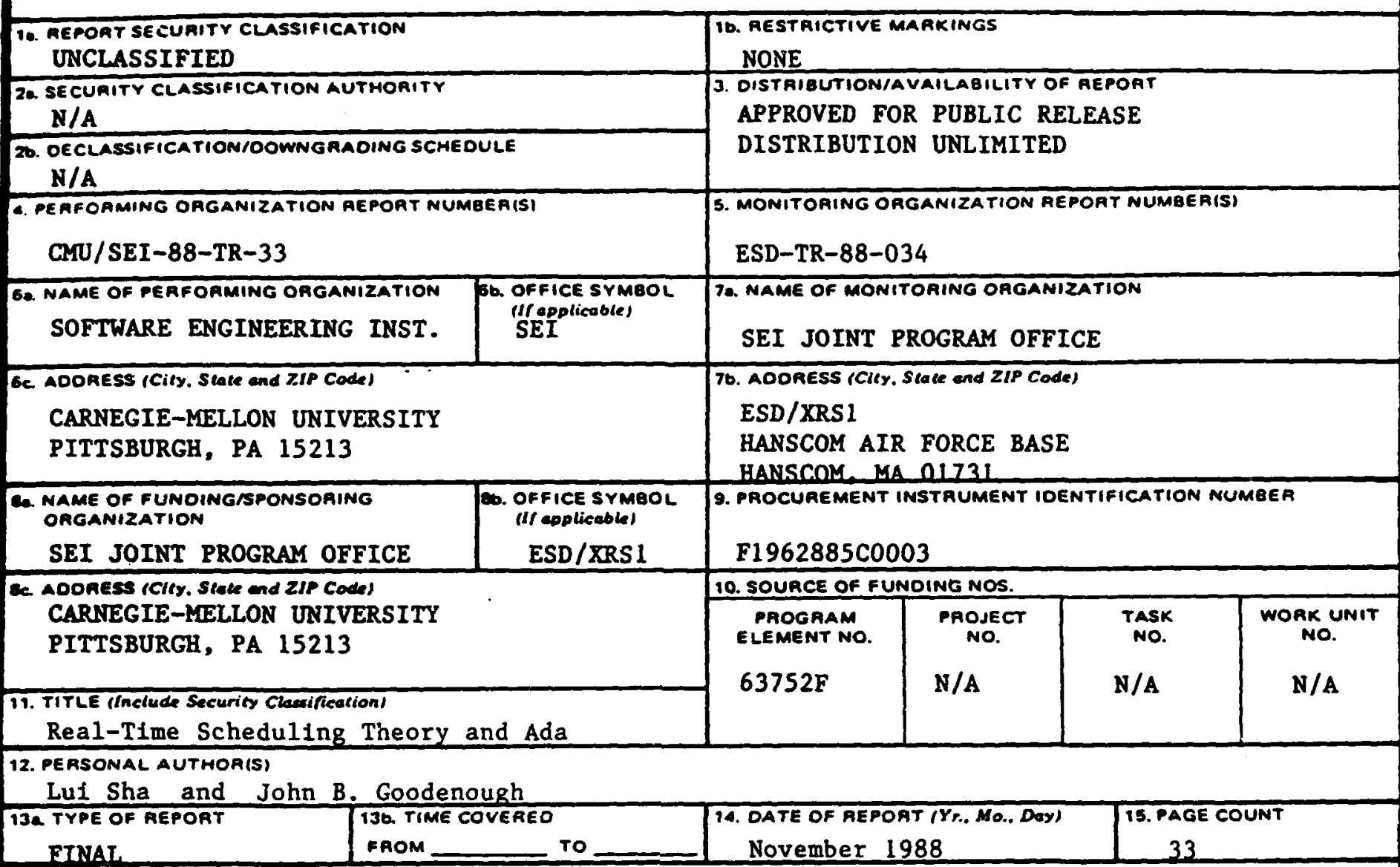

16. SUPPLEMENTAAY NOTATION

\begin{tabular}{|l|l|l|}
\hline 17. & COSATI CODES \\
\hline FIELO & GROUP & SUB. GR. \\
\hline & & \\
\hline & & \\
\hline
\end{tabular}

18. SUBJECT TERMS (Continue on reverse if necessory and identify by block numbert

Ada

Priority scheduling

Rea1-Time

Priority Inversion

Rate monotonic

19. AESTRACT (Continue on reverse i/ necestary and identify by block number)

The ada tasking model was intended to facilitate the management of concurrency in a priority-driven scheduling environment. In this paper, we will review some important results of a priority-based scheduling theory, illustrate its applications with examples, disucsss its implications for the Ada tasking model, and suggest workarounds that permit us to implement analytical scheduling algorithms within the existing framework of Ada.

\begin{tabular}{|c|c|c|}
\hline 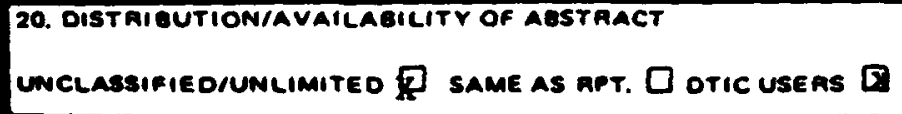 & $\begin{array}{l}\text { 21. AOSTRACT SECUAITY C } \\
\text { UNCLASSIFIED, UN }\end{array}$ & $\begin{array}{l}\text { CATION } \\
\text { ED DISTRIBUTION }\end{array}$ \\
\hline $\begin{array}{l}\text { 22. NAME OF AESPONSIELE INOIVIDUAL } \\
\text { KARL H. SHINGLER }\end{array}$ & $\begin{array}{l}\text { 22b. TELEPHONE NUMBEA } \\
\text { IInclude Area Codel } \\
412 \quad 268-7630\end{array}$ & $\begin{array}{l}\text { 22c. OFFICE SYMBOL } \\
\text { SEI JPO }\end{array}$ \\
\hline
\end{tabular}

